\title{
Mitos e verdades no ensino de técnicas projetivas
}

\author{
Fabiano Koich Miguel - Universidade Estadual de Londrina, Londrina, Paraná
}

\begin{abstract}
Resumo
O objetivo do presente estudo foi esclarecer mal entendidos comumente encontrados no ensino de técnicas projetivas. Foram desenvolvidos três temas: o mito da exclusividade da psicanálise sobre esses instrumentos, a reavaliação da nomenclatura das técnicas e o mito da ausência de validade. Encontrou-se que há publicações que dão suporte à utilização dessas técnicas por psicólogos de diversas abordagens, exemplificando com um teste desenvolvido pelo behaviorista Skinner. A possibilidade de uma nova terminologia, questionada por outros pesquisadores, foi apresentada. Além disso, a revisão concluiu que as técnicas demonstram cientificidade em diversos contextos da Psicologia.

Palavras chave: Avaliação psicológica; Técnicas projetivas; Validade.
\end{abstract}

Myths and facts in teaching of projective techniques

\begin{abstract}
The aim of this study was to clarify misunderstandings commonly found in the teaching of projective techniques. Three themes were developed: the myth of psychoanalysis' exclusivity on these instruments, the revaluation of the naming of the techniques, and the myth of lack of validity. It was found that there are publications supporting the use of such techniques by psychologists of various approaches, exemplifying with a test developed by behaviorist Skinner. The possibility of a new terminology, questioned by other researchers, was presented. In addition, the review concluded that the techniques demonstrated scientific criteria in various contexts of Psychology.

Keywords: Psychological assessment; Projective techniques; Validity.
\end{abstract}

Mitos y verdades en la enseñanza de técnicas proyectivas

\section{Resumen}

El objetivo de este estudio fue aclarar los malentendidos que se encuentran comúnmente en la enseñanza de las técnicas proyectivas. Tres temas fueron desarrollados: el mito de la exclusividad del psicoanálisis sobre los instrumentos, la reevaluación de la nomenclatura de las técnicas, y el mito de la ausencia de validez. Se encontró que hay publicaciones que apoyan el uso de esas técnicas por los psicólogos de diversos enfoques, ejemplificando con una prueba desarrollada por el behaviorista Skinner. La posibilidad de una nueva terminología, cuestionada por otros investigadores, fue presentada. Además, la revisión concluyó que las técnicas demuestran cientificidad en los diversos contextos de la psicología.

Palabras clave: Evaluación psicológica; Técnicas proyectivas; Validez.

\section{Introdução}

O início do século XXI foi marcado por um período de transformações na área de avaliação psicólogica, em que se enfrentava o problema da utilização inadequada de diversos testes e instrumentos (Noronha e cols., 2002; Primi, 2010; Wechsler, 2012). Vários resultados surgiram das ações tomadas naquele momento com o objetivo de reverter a precária situação então vigente, como debates, eventos científicos, aumento do número de pesquisas publicadas em periódicos reconhecidos, entre outros. No meio dessas ações, o Conselho Federal de Psicologia publicou a resolução $2 / 2003$, que definiu o que são testes psicológicos e estabeleceu critérios mínimos para utilização profissional dos mesmos (Anache \& Corrêa, 2010; CFP, 2003). Ainda, o CFP estabeleceu o ano temático de 2011-2012 como Ano da Avaliação Psicológica, promovendo atividades voltadas a discussão, reflexão e divulgação da área, além de publicações esclarecedoras nos conselhos regionais.

Apesar desse movimento e as mudanças alcançadas especialmente no âmbito científico, diversos autores ainda demonstram preocupação com a formação do psicólogo em avaliação psicológica durante a graduação (Bandeira, 2011; Castro, 2001; Fonseca, 2011; Löhr, 2011; Noronha, Carvalho, Miguel, Souza, \& Santos, 2010; Nunes e cols., 2012). Entre as dificuldades elencadas, pode-se destacar o ensino por professores com pouca qualificação na área e disciplinas com duração insuficiente, resultando em uma formação mecanicista e pouco aprofundada da avaliação e da aplicação de testes. Soma-se a isso a visão leiga sobre os instrumentos psicológicos, por vezes equivocadamente divulgados como formas de acesso aos segredos mais íntimos das pessoas, ou ferramentas capazes de descrever 
um sujeito por completo. Exemplos de matérias em revistas incluem, entre outros abusos, apresentar interpretações falsas para formas de avaliação, fazer críticas superficiais ou parciais sobre a validade e o contexto de aplicação, e até estampar manchas do Rorschach na revista Superinteressante (Garattoni, 2009; Manso, 2012) ou em artigos da Wikipedia.

Diante desse problema ainda presente e continuamente sendo abordado por pesquisadores da área, o objetivo deste artigo é esclarecer algumas questões potencialmente mal compreendidas e que costumam aparecer durante o ensino na graduação por meio de uma revisão da literatura relacionada ao tema. $\mathrm{O}$ foco será nas técnicas projetivas, que formam um grupo dentro dos procedimentos de avaliação psicológica. De acordo com a experiência no ensino de avaliação psicológica do autor, três mitos mais frequentes serão analisados: a exclusividade da psicanálise sobre as técnicas, a nomenclatura dos testes e a sua falta de validade.

\section{Definição de técnicas projetivas}

As técnicas projetivas se caracterizam pela apresentação de estímulos pouco estruturados, o que permite uma ampla variedade de respostas, maior foco nos aspectos qualitativos do desempenho e uma maior interação do psicólogo com o avaliando (Fensterseifer \& Werlang, 2008). O seu ensino na graduação se justifica, portanto, para além de simplesmente serem testes psicológicos, mas como exercício da observação e interação clínica.

Comumente associa-se a utilização de técnicas projetivas a psicólogos de orientação psicanalítica ou derivadas desta, como instrumentos destinados a avaliar aspectos inconscientes (Chabert, 2004; Fensterseifer \& Werlang, 2008; D. P. Schultz \& Schultz, 2011; Telles, 2000). Essa interpretação provavelmente se mantém devido ao próprio nome das técnicas, provindo do mecanismo de defesa do ego chamado projeção. A primeira menção da situação de projetar no mundo externo ideias incompatíveis com o ego foi proposta em 1895 por Freud (1950/2006a) em uma carta a Fliess sobre paranoia. O conceito desse mecanismo foi desenvolvido em outros momentos de sua obra até a proposta final, em "Totem e Tabu” (Freud, 1913/2006b), de que a projeção não é utilizada unicamente em situações onde há conflito, mas sim que é um mecanismo normal que faz com que a percepção de mundo seja alterada de acordo com as vivências afetivas anteriores, sejam elas agradáveis ou não.
A criação do termo "técnicas projetivas" deve-se a Frank (1939), ao analisar os testes para avaliação da personalidade disponíveis então - associação de palavras de Jung, Rorschach e TAT - e propor que o mecanismo de projeção estava subjacente à atividade, e que essas técnicas permitiam uma apreciação dinâmica e global do indivíduo. Desde aquela época até hoje, um corpo grande de publicações e pesquisas com esses instrumentos por parte de psicólogos de orientação psicanalítica possivelmente explique a associação comum entre os instrumentos e a abordagem. Nesse sentido, podem ser encontradas na literatura sugestões de que o psicólogo seja coerente com a fundamentação teórica do instrumento e não utilize técnicas projetivas se segue abordagens diferentes da psicanálise (Chabert, 2004; Keefe, Kopel, \& Gordon, 1980; Oliveira, Noronha, \& Dantas, 2006).

Essa interpretação pode ser um pouco precipitada. Sugere-se aqui que o mais apropriado seria entender a existência de um fenômeno (a resposta do indivíduo ao teste) e a compreensão desse fenômeno por meio de uma abordagem teórica. Dessa maneira, se excluiria a noção equivocada de que, quando uma abordagem explica o desempenho num teste, ela "toma para si" o construto, impedindo que outras teorias compreendam aquela situação de acordo com seus princípios. Na verdade, psicólogos de outras abordagens têm estudado o que acontece na situação de testagem. Um exemplo marcante é Skinner, um dos principais representantes do behaviorismo, apresentado a seguir.

\section{O uso de técnicas projetivas em outras abordagens}

$\mathrm{Na}$ metade da década de 1930, Skinner (1936) desenvolveu uma técnica para estudo de comportamentos verbais latentes. Em sua autobiografia, o autor explicou que se encontrava fechado em seu laboratório numa bela manhã de domingo e que, após algum tempo, o barulho persistente de um mecanismo parecia estar repetindo "youll never get out" (você jamais sairá). Considerando que esse fenômeno tinha importância "no sentido freudiano" e comparando-o a "borrões de tinta auditivos" (Skinner, 1979, p. 175), ele foi incentivado por Murray - que na época estava desenvolvendo o TAT (Rutherford, 2003) - a criar o que foi chamado de somador verbal. Esse teste reproduzia sequências de sílabas sem sentido, narradas pelo próprio Skinner ${ }^{1}$. Segundo o autor, após certo número de repetições,

\footnotetext{
${ }^{1}$ No website da B. F. Skinner Foundation (www.bfskinner.org) é possível fazer o download de uma faixa do Somador Verbal.
}

Psico-USF, Bragança Paulista, v. 19, n. 1, p. 97-106, jan./ abril 2014 
as pessoas começavam a identificar palavras naqueles sons. Ao sujeito, essas palavras poderiam parecer apenas repetições do estímulo auditivo, mas as variáveis que as originaram, mesmo não presentes em seu ambiente próximo e no momento da avaliação, poderiam ser encontradas na sua história de vida (Skinner, 1953/2003).

Em um tópico chamado "A ausência de autoconhecimento", Skinner (1953/2003) justificou que esse fenômeno é o mesmo que ocorre na utilização de testes projetivos no diagnóstico clínico, ou seja, permite revelar as variáveis desconhecidas pelo sujeito. $\mathrm{O}$ autor explicou, embasado em sua teoria, como um indivíduo pode desconhecer que está fazendo ou que está tendendo a fazer algo, ou até mesmo desconhecer as variáveis que influenciam no seu comportamento.

Nas décadas seguintes, algumas pesquisas foram feitas por outros autores com o Somador Verbal, posteriormente renomeado para Tautofone, contudo o instrumento acabou desaparecendo do rol de testes para avaliação da personalidade (Burns, Heiby, \& Tharp, 1983; Catania, 2008; Grings, 1942; Impellizzeri, 1970; Morris, 1978; Shakow \& Resenzweig, 1940; Trussell, 1939). Skinner, contudo, ainda demonstrou interesse nas técnicas de avaliação, inclusive sendo avaliado pelo Rorschach e TAT em 1953, como parte de uma pesquisa com diversos cientistas, com a condição de que seus nomes fossem revelados apenas após a morte (Grønnerød, Overskeid, \& Hartmann, 2012). Ele produziu um número surpreendente de 196 respostas no Rorschach.

Outros autores behavioristas fizeram considerações quanto ao uso de técnicas para avaliação comportamental, com os mesmos questionamentos quanto aos benefícios e limites encontrados na literatura de avaliação psicológica. Algumas dessas técnicas, como o uso da fantasia na produção verbal ou de desenhos, têm um procedimento muito semelhante ao que outras abordagens chamariam de "projetivas" (Banaco, 1997; Regra, 1997, 1999).

Além do behaviorismo, podem ser encontradas publicações de autores que sugerem outras abordagens para compreensão do fenômeno que ocorre na resposta aos testes. Como exemplo, pode-se citar o trabalho de Bunchaft e Vasoncellos (1998) com análise transacional; a proposta da análise fenômeno-estrutural do TAT e Roschach (Antúnez \& Amparo, 2008a; Antúnez \& Santoantonio, 2008b); a inclusão de aspectos cognitivos na interpretação do teste (Cattell, 1978; Exner Jr., 1989; Exner Jr. \& Sendín, 1999); como ferramentas na avaliação neuropsicológica (Vieira, Fay, \& Neiva-Silva, 2007); e até o foco em aspectos da percepção no TAT (Telles, 2000) e no Rorschach (Meyer, Viglione, Mihura, Erard, \& Erdberg, 2011), permitindo sua interpretação pelas diversas abordagens na psicologia.

\section{$A$ adequação do termo "projetivas"}

Um possível passo para a desvinculação das técnicas projetivas de apenas uma abordagem e utilização por outras na psicologia seria a adoção de uma nova nomenclatura. Meyer e Kurtz (2006) propuseram substituir por técnicas de desempenho, de resposta livre, expressivas, implícitas, a fim de evitar a má interpretação de termos não muito claros, como testes "objetivos" e "psicométricos" - englobando os inventários de personalidade - opostos aos "projetivos".

O termo "objetivos" pode gerar dois problemas de compreensão. Primeiro, pode levar à falsa noção de objetividade e segurança dos escores obtidos, quando na verdade os inventários dependem grandemente de uma adequada percepção do indivíduo sobre si mesmo, o que pesquisas vêm demonstrando que nem sempre ocorre (Cousineau \& Shedler, 2006; Shedler, Mayman, \& Manis, 1993). Segundo, as técnicas projetivas automaticamente seriam localizadas no outro extremo, ou seja, como não objetivas ou subjetivas, uma atribuição comum (Pasquali, 2001; Villemor-Amaral \& Pasqualini-Casado, 2006; Wood, Garb, Lilienfeld, \& Nezworski, 2002) que não condiz com a realidade. Alguns sistemas foram desenvolvidos a fim de evitar a variabilidade de interpretações entre psicólogos, permitindo procedimentos claros e inequívocos de codificação e transformação do desempenho no teste em números, como o sistema compreensivo do Rorschach e Zulliger ou a frequência de cores no Pfister.

Nesse mesmo sentido, os manuais apresentam estudos de precisão entre juízes, ou seja, o grau de concordância entre psicólogos avaliando os mesmos protocolos independentemente. Os resultados demonstram índices elevados, refutando a hipótese de que a própria subjetividade do psicólogo conduza a resultados discrepantes ou absurdos. Obviamente, as técnicas projetivas não são instrumento simples, sendo necessária uma formação sólida para que se utilizem corretamente os códigos de interpretação.

Além disso, a oposição de "psicométricos" a "projetivos" também pode gerar má interpretação das técnicas. Se teste psicométrico é baseado em teorias da medida e em normas estatísticas, então nestes poderiam 
ser incluídos o Pfister, Rorschach e Zulliger, uma vez que são pontuados e os resultados interpretados a partir de tabelas normativas.

\section{A validade das técnicas projetivas}

No que diz respeito à validade, ou seja, o quanto os instrumentos são capazes de medir aquilo a que se propõem, Villemor-Amaral e Werlang (2008) consideram que é possível observar dois polos de julgamento: a supervalorização e a desconfiança. Ambos podem ser prejudiciais para a utilização das técnicas. O primeiro caso pode acarretar na utilização sem crítica das ferramentas, confiando excessivamente no resultado do teste e prestando menos atenção a outras fontes de informação igualmente ou mais importantes.

Já no segundo caso, é possível encontrar tanto literaturas acadêmicas (D. P. Schultz \& Schultz, 2011; Wood e cols., 2002) quanto leigas (Garattoni, 2009; Lilienfeld, Wood, Garb, \& Wagenaar, 2005; Manso, 2012) afirmando que têm nenhuma ou pouca validade. Referindo-se especificamente à técnica de Rorschach, Mihura, Meyer, Dumitrascu e Bombel (2013) rebateram essas críticas, considerando que o fato de certo número de pesquisas apontarem para resultados diversificados pode ter levado críticos a simplesmente considerarem a técnica como inválida, o que pode ser uma interpretação apressada. Os autores realizaram uma extensa meta-análise, englobando 2.467 publicações de 1974 a 2011, encontrando evidências de validade para diversas variáveis do teste.

No âmbito nacional, a extensa produção científica com técnicas projetivas tem apresentado estudos que atestam as adequadas características psicométricas de validade e precisão dos instrumentos. Além disso, é possível encontrar pesquisas avaliando características psicológicas que vão além das tradicionais correlações entre construtos semelhantes, incluindo aspectos de vida importantes para o trabalho do psicólogo. Por exemplo, existem estudos com as Pirâmides Coloridas de Pfister para avaliação de alcoolismo (Villemor-Amaral, Silva, \& Primi, 2003), compulsão alimentar (Machado, Zilberstein, Cecconello, \& Monteiro, 2008), depressão (Villemor-Amaral, Primi, e cols., 2004), esquizofrenia (Villemor-Amaral e cols., 2005), estresse ocupacional de policiais militares (Aguiar, 2007), funções executivas em idosos (Formighieri, 2007), ideação suicida (Patutti, 2004), irritabilidade e agressividade em motoristas (Tawamoto \& Capitão, 2010), organização cognitiva de crianças surdas (Cardoso \& Capitão, 2007), toxicomania (Franco \& Villemor-Amaral, 2012), transtorno dissociativo de identidade (Faria, 2008), transtorno do pânico (Villemor-Amaral, Farah, \& Primi, 2004), entre outros.

Nesse mesmo sentido, as pesquisas com o teste das manchas de tinta de Zulliger, tanto no sistema compreensivo quanto no Klopfer, incluem avaliação da afetividade de pessoas surdas (Angelini \& Oliveira, 2003), afetividade em síndrome de Down (Rodrigues \& Alchieri, 2009), características emocionais na síndrome pré-menstrual (Montes \& Vaz, 2003), controle emocional de motoristas de transporte público (Rodriguez, 2009), depressão (Villemor-Amaral \& Machado, 2011), desempenho organizacional (Ferreira \& Villemor-Amaral, 2005), habilidades sociais (Grazziotin \& Scortegagna, 2012), inteligência (Candiani, Souza, Camilo, \& Candiani, 2003), toxicomania (Franco \& Villemor-Amaral, 2012), entre diversas outras.

O TAT apresenta pesquisas com avaliação de abuso sexual (Serafim, Saffi, Achá, \& Barros, 2011), afetividade na obesidade (Mishima \& Barbieri, 2009), aspectos emocionais de mulheres em hemodiálise (Medeiros, 2007), aspectos psicológicos na recidiva do câncer de mama (Peres, 2008), características dos casamentos duradouros (Paiva \& Gomes, 2006), desejo materno antes e após nascimento do filho (Barros, 2010), impacto de atributos físicos em decisões de recursos humanos (Souza, 2010), preocupação com desempenho sexual de doentes cardíacos (Lima, 2009), entre outras.

Alguns exemplos de estudos com Rorschach incluem avaliação de abuso sexual infantil (Scortegagna \& Villemor-Amaral, 2009), adolescentes homicidas (Resende, 2011), autismo (Araújo, Nascimento, \& Assumpção Jr., 2011), bulimia e anorexia nervosas (Oliveira-Cardoso \& Santos, 2012), compulsão alimentar (Passos, Yazigi, \& Claudino, 2008), esquizofrenia (Resende \& Argimon, 2012), gerentes de alto desempenho (Santos, 2009), inteligência emocional (Miguel \& Amaro, 2012), pacientes em tratamento de obesidade (Pinto, 2011), efetividade de terapia psicodinâmica (Yazigi, Amaro, Fiore, \& Semer, 2010), síndrome de Tourette (Bastos \& Vaz, 2009), tentativas de suicídio clinicamente graves (Vaz, 2010), transtorno do pânico (Castro, 2012), entre muitos outros.

Os exemplos citados não compõem a totalidade de pesquisas realizadas nos últimos anos, mas sim um recorte de algumas entre diversas características psicológicas passíveis de serem avaliadas e estudadas por meio das técnicas. Portanto, afirmar que não possuem validade é ignorar uma extensa produção científica. 
Logicamente, deve-se atentar para o contexto e os limites da aplicação, como deveria ser com qualquer instrumento de avaliação psicológica.

\section{Considerações finais}

O objetivo desse artigo foi esclarecer alguns mal-entendidos que costumam aparecer durante o ensino de técnicas projetivas na graduação. Foi empregado o método de revisão da literatura nacional a partir de 2003, focando nas técnicas projetivas de Pfister, Zulliger, TAT e Rorschach, sendo que, no caso deste último, dada a extensa produção, foi feito um recorte das publicações desde 2008. Os estudos mostraram que esses instrumentos têm demonstrado cientificidade na avaliação de características psicológicas em diversos contextos. Deve-se ressaltar que a literatura pesquisada não representa a totalidade de pesquisas disponíveis. Pretendeu-se, ao referenciar estudos com temas tão diversificados - por exemplo, transtornos alimentares, transtorno dissociativo de identidade, síndrome pré-menstrual, câncer de mama, entre outros - dar uma amostra da ampla gama de contextos em que o psicólogo pode atuar e contar com estudos de instrumentos para avaliação.

Ainda, foi feito levantamento de referências sugerindo que as técnicas projetivas podem ser utilizadas por psicólogos de orientação teórica diferente da psicanálise. Em específico, a aversão de alguns behavioristas à utilização de testes para avaliação, especialmente técnicas projetivas, parece incongruente, uma vez que o próprio Skinner demonstrou aceitação a esses instrumentos, tendo inclusive desenvolvido um próprio.

Este artigo não teve o propósito de desconsiderar as contribuições da psicanálise para as técnicas projetivas, que certamente possuem valor tanto histórico quanto contemporâneo, com publicações atuais (Chabert, 2004; Fensterseifer \& Werlang, 2008; Formiga \& Mello, 2000; Werlang \& Macedo, 2008). Muitas das pesquisas citadas no tópico sobre validade foram realizadas seguindo uma abordagem psicanalítica.

Também não houve intenção de comparar as teorias de Freud e Skinner. Para considerações nesse sentido, sugere-se a leitura de Grønnerød e cols. (2012) e Overskeid (2007). Contudo, teve a pretensão de se juntar ao grupo de autores que sugerem a possibilidade de utilização dessas preciosas formas de avaliação por outras abordagens, com o objetivo de ampliar os recursos científicos disponíveis aos psicólogos.
Em relação à possibilidade de utilização de uma nomenclatura mais adequada, existem sugestões na literatura, mas ainda não se chegou a um consenso quanto ao melhor termo. No Brasil, é possível encontrar autores empregando o termo "impressionistas" (Pasquali, 2001), "intuitivas" (Cunha, 2000) ou "autoexpressivas" (Villemor-Amaral \& Primi, 2012), contudo ainda junto de "projetivas". Sugere-se aqui a convergência para termos como "autoexpressivas" ou "técnicas de resposta livre", por aparentarem maior clareza quanto ao significado e possibilidade de adequação em diversas abordagens.

Como sugestão de futuros estudos, recomenda-se a exploração e divulgação de outros mitos que a presente pesquisa não teve a oportunidade de analisar. Como sugestões, pode-se citar o grau de influência que o conhecimento prévio dos estímulos das técnicas projetivas - seja pela internet ou outros meios de divulgação - pode ter na avaliação. Esse questionamento já foi iniciado por Schultz e Loving (2012), que encontraram informações potencialmente prejudiciais para usuários e psicólogos com a divulgação das manchas do Rorschach. Considerando-se as técnicas individualmente, outros mitos também podem ser encontrados e necessitam ser analisados. Por exemplo, Parada e Barbieri (2011) relataram experiências clínicas para demonstrar que o fato das pranchas do TAT remeterem a épocas mais antigas não dificulta a mobilização afetiva e as associações que surgem durante o desenvolvimento das histórias. Porém, as autoras evidenciaram a necessidade de pesquisas que abordem esse questionamento em participantes de várias idades.

\section{Referências}

Aguiar, F. L. S. (2007). Estresse ocupacional: contribuições das Pirâmides Coloridas de Pfister no contexto policial militar. Dissertação de Mestrado, Programa de Pós-Graduação em Psicologia, Universidade Federal do Pará, Belém.

Anache, A. A., \& Corrêa, F. B. (2010). As políticas do Conselho Federal de Psicologia para a avaliação psicológica. Em Conselho Federal de Psicologia (Org.), Avaliação psicológica: diretrizes na regulamentação da profissão (pp. 19-30). Brasília.

Angelini, S. N., \& Oliveira, R. V. (2003). Aplicação do teste verbal Zulliger (forma individual) em pessoas surdas. Psic: Revista de Psicologia da Vetor Editora, 4(1), 82-93. 
Antúnez, A. E. A., \& Amparo, D. M. (2008a). A abordagem fenômeno-estrutural e o método de Rorschach. Em A. E. Villemor-Amaral \& B. S. G. Werlang (Orgs.), Atualizações em métodos projetivos para avaliação psicológica (pp. 55-68). São Paulo: Casa do Psicólogo.

Antúnez, A. E. A., \& Santoantonio, J. (2008). Análise fenômeno-estrutural e o estudo de casos. Boletim Academia Paulista de Psicologia, 28(1), 53-71.

Araújo, C. A., Nascimento, R. S. G. F., \& Assumpção Jr., F. B. (2011). Autismo e psicodiagnóstico de Rorschach. Psico (Porto Alegre), 42(4), 434-441.

Banaco, R. A. (1997). Fantasia como instrumento de diagnóstico e tratamento: a visão de um behaviorista radical. Em M. Delitti (Org.), Sobre comportamento e cognição, volume 2: a prática da análise do comportamento e da terapia cognitivo-comportamental (pp. 115-119). São Paulo: Arbytes.

Bandeira, D. R. (2011). Repensando a formação em avaliação psicológica no Brasil. Em Conselho Federal de Psicologia (Org.), Ano da avaliação psicológica: textos geradores (pp. 129-132). Brasília.

Barros, I. P. M. (2010). Movimentos do desejo materno antes e após o nascimento do filho: um estudo longitudinal. Tese de Doutorado, Programa de Pós-Graduação em Psicologia, Universidade de São Paulo, São Paulo.

Bastos, A. G., \& Vaz, C. E. (2009). Estudo correlacional entre neuroimagem e a técnica de Rorschach em crianças com síndrome de Tourette. Avaliação Psicológica, 8(2), 229-244.

Bunchaft, G., \& Vasconcellos, V. L. P. (1998). Os testes projetivos em uma perspectiva não psicanalítica. Psicologia Clínica, Pós-Graduação e Pesquisa, 10, 7-23.

Burns, C. E. S., Heiby, E. M., \& Tharp, R. G. (1983). A verbal behavior analysis of auditory hallucinations. The Behavior Analyst, 6(2), 133-143.

Candiani, D. M. A., Souza, A. M. R., Camilo, D., \& Candiani, T. M. (2003). Estudo da validade de um método projetivo - Teste de Zulliger - por meio de parâmetros psicométricos. Psic: Revista de Psicologia da Vetor Editora, 4(2), 36-43.

Cardoso, L. M., \& Capitão, C. G. (2007). Avaliação psicológica de crianças surdas pelo Teste das Pirâmides Coloridas de Pfister. Psico-USF, 12(2), 135-144.
Castro, P. F. (2001). O ensino do Rorschach em uma amostra brasileira. Psicologia: Ciência e Profissão, 21(1), 46-53. doi: 10.1590/S1414-98932001000100006

Castro, P. F. (2012). Indicadores de comorbidade em pacientes com transtorno de pânico avaliados pelo método de Rorschach. Temas em Psicologia, 20(2), 537-554. doi: 10.9788/TP2012.2-19

Catania, A. C. (2008). Skinner's Verbal Behavior in a new century. International Journal of Psychology and Psychological Therapy, 8(3), 277-285.

Cattell, R. B. (1978). Princípios de esquemas nos testes "projetivos" ou de má percepção da personalidade. Em H. H. Anderson \& G. L. Anderson (Orgs.), Técnicas projetivas do diagnóstico psicológico (pp. 69-111). São Paulo: Mestre Jou.

CFP - Conselho Federal de Psicologia. (2003). Resolução $n^{\circ}$ 02/2003. Brasília: Conselho Federal de Psicologia.

Chabert, C. (2004). Psicanálise e métodos projetivos. São Paulo: Vetor.

Cousineau, T. M., \& Shedler, J. (2006). Predicting physical health: Implicit mental health measures versus self-report scales. Journal of Nervous \& Mental Disease, 194(6), 427-432. doi: 10.1097/01. nmd.0000221373.74045.51

Cunha, J. A. (2000). Psicodiagnóstico-V. Porto Alegre: Artmed.

Exner Jr., J. E. (1989). Searching for projection in the Rorschach. Journal of Personality Assessment, 53(3), 520-536.

Exner Jr., J. E., \& Sendín, C. (1999). Manual de interpretação do Rorschach para o sistema compreensivo. São Paulo: Casa do Psicólogo.

Faria, M. A. (2008). O teste de Pfister e o transtorno dissociativo de identidade. Avaliação Psicológica, 7(3), 359-370.

Fensterseifer, L., \& Werlang, B. S. G. (2008). Apontamentos sobre o status científico das técnicas projetivas. Em A. E. Villemor-Amaral \& B. S. G. Werlang (Orgs.), Atualizações em métodos projetivos para avaliação psicológica (pp. 15-33). São Paulo: Casa do Psicólogo.

Ferreira, M. E. A., \& Villemor-Amaral, A. E. (2005). O teste de Zulliger e avaliação de Psico-USF, Bragança Paulista, v. 19, n. 1, p. 97-106, jan./abril 2014 
desempenho. Paidéia, 15(32), 367-376. doi: 10.1590/S0103-863X2005000300006

Fonseca, C. M. S. M. S. (2011). Avaliação psicológica e suas vicissitudes: a formação do psicólogo como foco. Em Conselho Federal de Psicologia (Org.), Ano da avaliação psicológica: Textos geradores (pp. 133138). Brasília.

Formiga, N. S., \& Mello, I. (2000). Testes psicológicos e técnicas projetivas: uma integração para um desenvolvimento da interação interpretativa indivíduo-psicólogo. Psicologia: Ciência e Profissão, 20(2), 12-19. doi: 10.1590/S1414-98932000000200004

Formighieri, M. S. B. (2007). Afetividade e funções executivas em idosos: estudo normativo com Wisconsin Card Sorting Test e Pfister. Dissertação de Mestrado, Programa de Pós-Graduação em Psicologia, Universidade de São Paulo, Ribeirão Preto.

Franco, R. R. C., \& Villemor-Amaral, A. E. (2012). Validade incremental do Zulliger e do Pfister no contexto da toxicomania. Psico-USF, 17(1), 73-83. doi: 10.1590/S1413-82712012000100009

Frank, L. K. (1939). Projective methods for the study of personality. The Journal of Psychology, 8(2), 389413. doi: $10.1080 / 00223980.1939 .9917671$

Freud, S. (2006a). Rascunho H: Paranoia. Em Obras psicológias completas de Sigmund Freud: Edição standard brasileira (Vol. 1, pp. 253-258). Rio de Janeiro: Imago. (Original publicado em 1950).

Freud, S. (2006b). Totem e tabu. Em Obras psicológias completas de Sigmund Freud: Edição standard brasileira (Vol. 13, pp. 11-163). Rio de Janeiro: Imago. (Original publicado em 1913).

Garattoni, B. (2009, agosto). Manual secreto do RH: quais são, e como funcionam, os testes psicológicos usados nas entrevistas de emprego. Superinteressante, 268, 76-79.

Grazziotin, J. B. D., \& Scortegagna, S. A. (2012). Zulliger e habilidade social: Evidências de validade no contexto empresarial. Psicologia: Reflexão e Crítica, 25(1), 69-78. doi: 10.1590/ S0102-79722012000100009

Grings, W. W. (1942). The verbal summator technique and abnormal mental states. The Journal of Abnormal and Social Psychology, 37(4), 529-545. doi: 10.1037/ h0054337
Grønnerød, C., Overskeid, G., \& Hartmann, E. (2012). Under Skinner's skin: gauging a behaviorist from his Rorschach protocol. Journal of Personality Assessment, 95(1), 1-12. doi: 10.1080/00223891.2012.696081

Impellizzeri, I. (1970). Use of the verbal summator technique with language and nonlanguage majors in college. The Journal of General Psychology, 83(2), 143-149. doi: 10.1080/00221309.1970.9710796

Keefe, F. J., Kopel, S. A., \& Gordon, S. B. (1980). Manual prático de avaliação comportamental. São Paulo: Manole.

Lilienfeld, S. O., Wood, J. M., Garb, H. N., \& Wagenaar, J. (2005). What's wrong with this picture? Scientific American, 16, 50-57. doi: 10.1038/ scientificamericanmind0405-50

Lima, V. M. (2009). Atividade sexual dos pacientes submetidos à cirurgia de valvopatia. Dissertação de Mestrado, Faculdade de Medicina, Universidade de São Paulo, São Paulo.

Löhr, S. S. (2011). Avaliação psicológica na formação do profissional da Psicologia: algumas reflexões. Em Conselho Federal de Psicologia (Org.), Ano da avaliação psicológica: textos geradores (pp. 143-149). Brasília.

Machado, C. E., Zilberstein, B., Cecconello, I., \& Monteiro, M. (2008). Compulsão alimentar antes e após a cirurgia bariátrica. Arquivos Brasileiros de Cirurgia Digestiva, 21(4), 185-191. doi: 10.1590/ S0102-67202008000400007

Manso, U. A. (2012, julho-agosto-setembro). O estranho mundo da seleção. Você RH, 22, 32-37.

Medeiros, G. A. (2007). Gestação em mulheres em tratamento hemodialítico: repercussões do adoecimento sobre o desejo pela maternidade. Dissertação de Mestrado, Faculdade de Medicina, Universidade de São Paulo, São Paulo.

Meyer, G. J., \& Kurtz, J. E. (2006). Advancing personality assessment terminology: time to retire "objective" and "projective" as personality test fescriptors. Journal of Personality Assessment, 87(3), 223-225. doi: 10.1207/s15327752jpa8703_01

Meyer, G. J., Viglione, D. J., Mihura, J. L., Erard, R. E., \& Erdberg, P. (2011). Rorschach Performance Assessment System: administration, coding, interpretation, and technical manual. Toledo: Rorschach Performance Assessment System. 
Miguel, F. K., \& Amaro, M. C. P. (2012). Relação do Rorschach com percepção de emoções. Em D. M. Amparo, E. T. K. Okino, C. L. C. Hisatugo, F. L. Osório \& M. Tavares (Orgs.), Livro de programa e resumos do VI Congresso da Associação Brasileira de Rorschach e Métodos Projetivos (pp. 180). Brasília: ASBRo.

Mihura, J. L., Meyer, G. J., Dumitrascu, N., \& Bombel, G. (2013). The validity of individual Rorschach variables: systematic reviews and meta-analyses of the comprehensive system. Psychological Bulletin, 139(3), 548-605. doi: 10.1037/a0029406

Mishima, F. K. T., \& Barbieri, V. (2009). Saúde feminina: Considerações sobre psicodiagnóstico interventivo na obesidade. Mudanças - Psicologia da Saúde, 17(2), 92-100.

Montes, R. M., \& Vaz, C. E. (2003). Condições afetivo-emocionais em mulheres com síndrome pré-menstrual através do Z-Teste e do IDATE. Psicologia: Teoria e Pesquisa, 19(3), 261-267. doi: 10.1590/S0102-37722003000300008

Morris, W. W. (1978). Outros métodos projetivos. Em H. H. Anderson \& G. L. Anderson (Orgs.), Técnicas projetivas do diagnóstico psicológico (pp. 505-527). São Paulo: Mestre Jou.

Noronha, A. P. P., Carvalho, L. F., Miguel, F. K., Souza, M. S., \& Santos, M. A. (2010). Sobre o ensino de avaliação psicológica. Avaliação Psicológica, 9(1), 139-146.

Noronha, A. P. P., Ziviani, C., Hutz, C. S., Bandeira, D. R., Custódio, E. M., Alves, I. B., . . . Domingues, S. (2002). Em defesa da avaliação psicológica. Avaliação Psicológica, 1(2), 173-174.

Nunes, M. F. O., Muniz, M., Reppold, C. T., Faiad, C., Bueno, J. M. H., \& Noronha, A. P. P. (2012). Diretrizes para o ensino da avaliação psicológica. Avaliação Psicológica, 11(2), 309-316.

Oliveira-Cardoso, É. A., \& Santos, M. A. (2012). Avaliação psicológica de pacientes com anorexia e bulimia nervosa: indicadores do teste de Rorschach. Fractal: Revista de Psicologia, 24(1), 159-174.

Oliveira, K. L., Noronha, A. P. P., \& Dantas, M. A. (2006). Instrumentos psicológicos: Estudo comparativo entre estudantes e profissionais cognitivo-comportamentais. estudos de
Psicologia (Campinas), 23(4), 359-367. doi: 10.1590/ S0103-166X2006000400004.

Overskeid, G. (2007). Looking for Skinner and finding Freud. American Psychologist, 62(6), 590-595. doi: 10.1037/0003-066X.62.6.590.

Paiva, M. L. S. C., \& Gomes, I. C. (2006). Casamentos duradouros: o uso de entrevistas e o TAT na análise psicanalítica da relação conjugal. Mudanças - Psicologia da Saúde, 14(2), 151-159.

Parada, A. P., \& Barbieri, V. (2011). Reflexões sobre o uso clínico do TAT na contemporaneidade. Psico-USF, 16(1), 117-125. doi: 10.1590/ S1413-82712011000100013

Pasquali, L. (2001). Técnicas de exame psicológico - TEP: manual. São Paulo: Casa do Psicólogo.

Passos, T. C. B. M., Yazigi, L., \& Claudino, A. M. (2008). Aspectos ideativos no transtorno da compulsão alimentar periódica: estudo com o Rorschach. Psico-USF, 13(1), 69-74. doi: 10.1590/ S1413-82712008000100009.

Patutti, C. A. O. B. (2004). Transtorno de pânico e ideação suicida: características de personalidade por meio do teste de Pfister. Dissertação de Mestrado, Programa de Pós-Graduação da Faculdade de Ciências Médicas, Universidade Estadual de Campinas, Campinas.

Peres, R. S. (2008). Na trama do trauma: relacões entre a personalidade de mulheres acometidas por câncer de mama e a recidiva oncológica sob a ótica da psicossomática psicanalítica. Tese de Doutorado, Programa de PósGraduação em Psicologia, Universidade de São Paulo, Ribeirão Preto.

Pinto, L. K. (2011). Um estudo com o Psicodiagnóstico de Rorschach sobre o funcionamento psíquico de pacientes que realizaram tratamento para a obesidade. Dissertação de Mestrado, Programa de Pós-Graduação em Psicologia, Universidade de São Paulo, São Paulo.

Primi, R. (2010). Avaliação psicológica no Brasil: Fundamentos, situação atual e direções para o futuro. Psicologia: Teoria e Pesquisa, 26(especial), 25-35. doi: 10.1590/S0102-37722010000500003

Regra, J. A. G. (1997). Fantasia: instrumento de diagnóstico e tratamento. Em M. Delitti (Org.), Sobre comportamento e cognição, volume 2: a prática da análise do comportamento e da terapia cognitivo-comportamental (pp. 107-114). São Paulo: Arbytes.

Psico-USF, Bragança Paulista, v. 19, n. 1, p. 97-106, jan./ abril 2014 
Regra, J. A. G. (1999). A fantasia e o desenho. Em R. R. Kerbauy \& R. C. Wielenska (Orgs.), Sobre comportamento e cognição, volume 4: psicologia comportamental cognitiva - Da reflexão teórica à diversidade da aplicação (pp. 105-115). Santo André: Esetec.

Resende, A. C. (2011). A personalidade de adolescentes que cometeram homicídio por meio do método de Rorschach. Estudos (Goiânia), 38(1), 29-48.

Resende, A. C., \& Argimon, I. I. L. (2012). A técnica de Rorschach e os critérios da CID-10 para o diagnóstico da esquizofrenia. Psicologia: Reflexão e Crítica, 25(3), 422-434. doi: 10.1590/ S0102-79722012000300002.

Rodrigues, E. C., \& Alchieri, J. C. (2009). Avaliação das características de afetividade em crianças e jovens com síndrome de Down. Psico-USF, 14(1), 107116. doi: 10.1590/S1413-82712009000100011.

Rodriguez, S. Y. S. (2009). Capacidade de controle emocional e impulsos agressivos em motoristas do transporte coletivo público envolvidos em acidentes de trânsito. Dissertação de Mestrado, Programa de Pós-Graduação em Psicologia, Pontifícia Universidade Católica do Rio Grande do Sul, Porto Alegre.

Rutherford, A. (2003). B. F. Skinner and the Auditory Inkblot: the rise and fall of the Verbal Summator as a projective technique. History of Psychology, 6(4), 362-378. doi: 10.1037/1093-4510.6.4.362

Santos, S. C. G. (2009). Avaliação da personalidade de gerentes de alto desempenho por meio do método de Rorschach. Tese de doutorado, Programa de Pós-Graduação em Psicologia, Pontifícia Universidade Catolica do Rio Grande do Sul, Porto Alegre.

Schultz, D. P., \& Schultz, S. E. (2011). Teorias da personalidade. São Paulo: Cengage Learning.

Schultz, D. S., \& Loving, J. L. (2012). Challenges since Wikipedia: The availability of Rorschach information online and internet users' reactions to online media coverage of the Rorschach-Wikipedia debate. Journal of Personality Assessment, , 94(1), 73-81. doi: 10.1080/00223891.2011.627963

Scortegagna, S. A., \& Villemor-Amaral, A. E. (2009). Autopercepção no Rorschach de vítimas de abuso sexual infantil. Psico (Porto Alegre), 40(3), 328-336.

Serafim, A. P., Saffi, F., Achá, M. F. F., \& Barros, D. M. d. (2011). Dados demográficos, psicológicos e comportamentais de crianças e adolescentes vítimas de abuso sexual. Revista de Psiquiatria Clínica, 38(4), 143147. doi: 10.1590/S0101-60832011000400006.

Shakow, D., \& Resenzweig, S. (1940). The use of the Tautophone ("Verbal Summator") as an auditory apperceptive test for the study of personality. Journal of Personality, 8(3), 216-226. doi: 10.1111/ j.1467-6494.1940.tb02177.x.

Shedler, J., Mayman, M., \& Manis, M. (1993). The illusion of mental health. American Psychologist, 48(11), 1117-1131.

Skinner, B. F. (1936). The Verbal Summator and a method for the study of latent speech. The Journal of Psychology, 2(1), 71-107. doi: 10.1080/00223980.1936.9917445.

Skinner, B. F. (1979). The shaping of a behaviorist: part two of an autobiography. New York: Knopf.

Skinner, B. F. (2003). Ciência e comportamento humano. São Paulo: Martins Fontes. (Original publicado em 1953).

Souza, A. A. L. (2010). A influência de atributos físicos na formação de primeiras impressões: uso do TAT e impacto no processo decisório de profissionais de recursos humanos. Tese de Doutorado, Programa de Pós-Graduação em Psicologia, Universidade de São Paulo, São Paulo.

Tawamoto, J. M., \& Capitão, C. G. (2010). Evidências de validade do teste de Pfister: agressividade e irritabilidade em motoristas. Estudos Interdisciplinares em Psicologia, 1(1), 40-65.

Telles, V. S. (2000). A desvinculação do TAT do conceito de "projeção" e a ampliação de seu uso. Psicologia USP, 11(1), 63-83. doi: 10.1590/ S0103-65642000000100005.

Trussell, M. A. (1939). The diagnostic value of the verbal summator. The Journal of Abnormal and Social Psychology, 34(4), 533-538. doi: 10.1037/h0058202

Vaz, S. B. (2010). O método de Rorschach no estudo de casos de tentativa de suicídio clinicamente grave. Dissertação de Mestrado, Programa de Pós-Graduação em Psicologia Clínica e Cultura, Universidade de Brasília, Brasília.

Vieira, C., Fay, E. S. M., \& Neiva-Silva, L. (2007). Avaliação psicológica, neuropsicológica e recursos em neuroimagem: novas perspectivas em saúde mental. Aletheia, 26, 181-195. 
Villemor-Amaral, A. E., Farah, F. H. Z., \& Primi, R. (2004). O Teste das Pirâmides Coloridas e o transtorno do pânico. Psicologia em Estudo (Maringá), 9(2), 301-307. doi: 10.1590/S1413-73722004000200016.

Villemor-Amaral, A. E., \& Machado, M. A. S. (2011). Indicadores de depressão do Zulliger no Sistema Compreensivo (ZSC). Paidéia, 21(48), 21-27. doi: 10.1590/S0103-863X2011000100004.

Villemor-Amaral, A. E., \& Pasqualini-Casado, L. (2006). A cientificidade das técnicas projetivas em debate. Psico-USF, 11(2), 185-193. doi: 10.1590/ S1413-82712006000200007.

Villemor-Amaral, A. E., \& Primi, R. (2012). Teste de Zulliger no sistema compreensivo, ZSC: forma individual. São Paulo: Casa do Psicólogo.

Villemor-Amaral, A. E., Primi, R., Farah, F. H. Z., Silva, S. M., Cardoso, L. M., \& Franco, R. R. C. (2004). A depressão no Teste das Pirâmides Coloridas de Pfister. Paidéia, 14(28), 169-176. doi: 10.1590/ S0103-863X2004000200006.

Villemor-Amaral, A. E., Primi, R., Franco, R. R. C., Farah, F. H. Z., Cardoso, L. M., \& Silva, T. C. d. (2005). O teste de Pfister e sua contribuição para diagnóstico da esquizofrenia. Revista do Departamento de Psicologia - UFF, 17(2), 89-98. doi: 10.1590/ S0104-80232005000200008.

Villemor-Amaral, A. E., Silva, T. C., \& Primi, R. (2003). Indicadores de alcoolismo no Teste das Pirâmides
Coloridas de Max Pfister. Psico-USF, 8(1), 33-38. doi: 10.1590/S1413-82712003000100005.

Villemor-Amaral, A. E., \& Werlang, B. S. G. (2008). Apresentação. Em A. E. Villemor-Amaral \& B. S. G. Werlang (Orgs.), Atualizações em métodos projetivos para avaliação psicológica (pp. 11-13). São Paulo: Casa do Psicólogo.

Wechsler, S. M. (2012). Prefácio. Em R. B. F. Silva \& S. V. C. Areosa (Orgs.), Avaliação psicológica: desafios e possibilidades para a psicologia contemporânea (pp. 7-9). Santa Cruz do Sul: EDUNISC.

Werlang, B. S. G., \& Macedo, M. M. K. (2008). O teste dos contos de fadas. Em A. E. Villemor-Amaral \& B. S. G. Werlang (Orgs.), Atualizações em métodos projetivos para avaliação psicológica (pp. 183-192). São Paulo: Casa do Psicólogo.

Wood, J. M., Garb, H. N., Lilienfeld, S. O., \& Nezworski, M. T. (2002). Clinical assessment. Annual Review of Psychology, 53, 519-543. doi: 10.1146/annurev.psych.53.100901.135136.

Yazigi, L., Amaro, T. C., Fiore, M. L. M., \& Semer, N. L. (2010). Rorschach Sistema Compreensivo na avaliação de psicoterapia psicodinâmica. Psico-USF, 15(2), 257-270. doi: 10.1590/S1413-82712010000200013.

Recebido em: 05/02/2013

Reformulado em: 23/05/2013

Aprovado em: 18/09/2013

Sobre o autor:

Fabiano Koich Miguel possui graduação em Psicologia pela Universidade Presbiteriana Mackenzie (2002), concluiu mestrado (2006) e doutorado (2010) em Avaliação Psicológica pela Universidade São Francisco. Atualmente é professor adjunto da Universidade Estadual de Londrina (UEL).

Contato com o autor:

Departamento de Psicologia e Psicanálise - CCB - UEL

Campus Universitário - Caixa Postal 6001

Londrina-PR

86051-980

E-mail: fabiano@avalpsi.com.br 